Conclusions Short segment Barrett's can be difficult to assess accurately. In our analysis, cases that did not meet the criteria for Barrett's diagnosis on endoscopy, had segment $<1 \mathrm{~cm}$ or an atypical $\mathrm{Z}$ line.

We have shown a potential $49 \%$ reduction in unnecessary gastroscopies, which could result in avoidance of unnecessary health and procedural anxiety for patients, and cut waiting lists and associated costs.

In addition, we suggest that patients with previous suspected Barrett's should be evaluated on dedicated surveillance lists, by endoscopists with expertise in Barrett's assessment.

\section{PTU-53 EFFICACY AND SAFETY OF ENDOSCOPIC AMPULLECTOMY IN THE UK}

Sujith Sasidharan Nair*, Mohamed Abdelrahim, Lazaros Varytimiadis, Asma Al-Kandari, Patrick Goggin, Professor Pradeep Bhandari. Queen Alexandra Hospital,Portsmouth Hospital University Trust, Portsmouth, UK

\subsection{6/gutjnl-2021-BSG.126}

Introduction Endoscopic ampullectomy is a minimally invasive technique of treating non-invasive lesions involving the ampulla of Vater and offers an alternative to major surgical intervention. In this study, we describe the safety and outcome of this procedure from a single large tertiary unit in the UK.

Methods Data were prospectively collected on an electronic database. Parameters related to ampullectomy outcome and complications were retrospectively analyzed.

10 to $20 \mathrm{~mm}$ snare sizes were used in these patients. ENDOCUT Q effect 2 was used. All patients were given indomethacin suppositories. Submucosal injection was performed only where the ampullary lesions spilled over to the duodenal mucosa.

Results A total of 48 cases were included in this analysis, between 2009 and 2021. The mean age was 62.4 years. Female represented $55.3 \%$ of patients. Mean duration of follow up was 118.29 weeks (27 months). 29 (60.4\%) lesions were pure ampullary, and the remaining involved the duodenal wall as well. Lesions ranged from 5 to $80 \mathrm{~mm}$ in size, with average size of $22.2 \mathrm{~mm}$. 6 patients (12.5\%) had familial adenomatous polyposis FAP. Pre resection histology confirmed HGD in one patient (2\%), and neuro-endocrine tumor NET in one case (2\%). Post resection histology showed focal adenocarcinoma in 2 patients (4.1\%).

Prophylactic PD stenting was performed in 31 cases $(64.5 \%)$ of which one patient developed pancreatitis. Out of the 17 cases (35.4\%) who did not have PD stenting, none developed pancreatitis. Adjunctive APC used in 11 cases (22.9\%), and submucosal injection in 28 cases $(58.3 \%)$. Enbloc resection was achieved in 16 (33.3\%), while piecemeal in 32 cases $(66.6 \%)$. Recurrence observed in 12 out of 48 cases (25.0\%). Piecemeal resection was significantly associated with higher risk of recurrence $(\mathrm{P}=0.0404)$. Early bleeding (including intra-procedural and bleeding within 24 hours of procedure) happened in 6 patients $(12.5 \%)$, delayed bleeding (after 24 hours) in 2 cases (4.1\%), all were successfully treated endoscopically. Only 1 patient (2\%) had Pancreatitis, and one patient developed ampullary stenosis post procedure. There was no report of perforation, need for emergency surgery or 30-day mortality in this series.

Conclusion

- Endoscopic ampullectomy seems safe and effective in expert hands
- The recurrence rate observed in this series was 25\%, all of them treated with further endoscopic therapy

- Piecemeal resection was significantly associated with higher risk of recurrence, all managed with further endoscopic therapy

- If indomethacin suppositories are universally used, prophylactic pancreatic stenting may not be needed as the risk of pancreatitis is very low

\section{PTU-54 EARLY PROOF OF CONCEPT STUDY OF A NOVEL ULTRASONIC MEASUREMENT DEVICE FOR UPPER GI ENDOSCOPY}

${ }^{1,2}$ Israa Bondoqa*, ${ }^{1}$ Simon Toh, ${ }^{2}$ Raymond Lee, ${ }^{2}$ Martino Pani. ${ }^{1}$ Portsmouth Nhs Trust, Dept of Upper GI Surgery, Portsmouth, UK; ${ }^{2}$ University of Portsmouth, Faculty of Technology, Portsmouth, UK

\subsection{6/gutjnl-2021-BSG.127}

Introduction Measurement of the distance of an endoscope tip from mouth guard is estimated visually in the dark by the endoscopist. To improve the precision and ease of measurement, we developed a prototype device that attaches to any endoscope externally so that this measurement can be displayed on-screen in real-time for recording and review.

Methods A prototype ultrasonic measurement device that clamps to the endoscope outside the patient was devised and tested by an experienced and novice endoscopist with multiple repeated measurements made of a fixed $Z$ line at $39 \mathrm{~cm}$. Paired measurements were made with the endoscopist's own done by usual visual inspection. The device measures how much of the endoscope is outside the patient to calculate the distance of the scope tip within the patient. This study was performed in a Koken EGD simulator (GTSimluators, Davie, Florida, USA)

Results Fifteen paired measurements each were performed by both endoscopists with the results summarized in this table (Table 1.):

There were no significant differences in the measurements made by the device compared with either endoscopists. The device did not impede endoscopy in any way, The initial study guided improvements in the design and data processing to reduce variability.

Conclusions This early proof of concept feasibility study has encouraged us to develop a workable prototype device that we will test in a series of upper GI endoscopies in patients (ScopeMeasure Study IRAS Ref 20/SC/0387). This device will be compatible with any endoscope and will be reusable within a disposable sterile sleeve. We hope that automated

\begin{tabular}{llll} 
Abstract PTU-54 Table 1 & & \\
\hline & $\begin{array}{l}\text { Mean (Range) } \\
\mathrm{cm}\end{array}$ & $\begin{array}{l}\text { Median (1SD) } \\
\mathrm{cm}\end{array}$ & $\begin{array}{l}\text { Paired student t-test (2 } \\
\text { tailed) }\end{array}$ \\
\hline $\begin{array}{l}\text { Experienced endoscopist } \\
\text { results }\end{array}$ & $39.1(39-39.5)$ & 39 & $\mathrm{p}=0.183$ \\
$\begin{array}{l}\text { Device Results } \\
\text { Experienced endoscopist } \\
\text { results }\end{array}$ & $\begin{array}{l}30.1(37-46) \\
\text { Device Results }\end{array}$ & $39(2.5)$ & \\
\hline
\end{tabular}

